\title{
A Bayesian Classifier for X-Ray Pulsars Recognition
}

\author{
Hao Liang, Yafeng Zhan, and Chaowei Duan \\ School of Aerospace Engineering and Space Centre, Tsinghua University, Beijing 100084, China \\ Correspondence should be addressed to Yafeng Zhan; zhanyf@tsinghua.edu.cn
}

Received 18 June 2016; Revised 21 September 2016; Accepted 16 October 2016

Academic Editor: Paul Williams

Copyright (C) 2016 Hao Liang et al. This is an open access article distributed under the Creative Commons Attribution License, which permits unrestricted use, distribution, and reproduction in any medium, provided the original work is properly cited.

\begin{abstract}
Recognition for X-ray pulsars is important for the problem of spacecraft's attitude determination by X-ray Pulsar Navigation (XPNAV). By using the nonhomogeneous Poisson model of the received photons and the minimum recognition error criterion, a classifier based on the Bayesian theorem is proposed. For X-ray pulsars recognition with unknown Doppler frequency and initial phase, the features of every X-ray pulsar are extracted and the unknown parameters are estimated using the Maximum Likelihood (ML) method. Besides that, a method to recognize unknown X-ray pulsars or X-ray disturbances is proposed. Simulation results certificate the validity of the proposed Bayesian classifier.
\end{abstract}

\section{Introduction}

PULSARS have been recognized as nature's "most stable clock" $[1,2]$ and could emit ideal period pulse signals in multielectromagnetic bands [3]. Since Chester and Butman firstly proposed using X-ray pulsars for navigation [4], many scholars focus on this completely autonomous celestial navigation method [3,5-8]. Recognition for X-ray pulsars is one of the key technologies in the X-ray Pulsar Navigation (XPNAV) system. When XPNAV system is failure or restarted, the spacecraft rolls over and the attitude of the spacecraft is unknown. At this time, the detector receives one $\mathrm{X}$-ray pulsar signal in a very short time. If we recognize which $\mathrm{X}$-ray pulsar emits the signal, we can extract the observed pulsar's direction vector from the XPNAV Database [3] to speed up the spacecraft's attitude redetermination [6].

At present, most literatures proposed to recovery the X-ray pulsar's profile [9] and use transforming method to extract the features of profile for pulsars recognition, such as the Selected Line Spectra Transform [10, 11], the BispectraMellin Transform [12], the S Transform (ST) [13, 14], and the Wavelet Transform [15]. Besides that, the neural network has been used for pulsars recognition at the Pulsar Arecibo Lband Feed Array (PALFA) [16]. The major disadvantage of the above classifiers is that they have to recovery the X-ray pulsar's profile. As the X-ray pulsar is always far away from the solar system (thousands of light years), the received X-ray pulsar signal is very weak. Therefore, unless the observation time for X-ray pulsars could last hundreds of seconds, it is too hard to build the X-ray pulsar's profile well. In consequence, the existing classifiers are inefficient and even useless for the scenario with short observation time.

XPNAV could only use the well-known X-ray pulsars for navigation. The XPNAV Database stores each alternative $\mathrm{X}$-ray pulsar's prior knowledge, which includes the X-ray pulsar's direction vector, the X-ray background noise's rate, and the X-ray pulsar signal's flux, period, and standard profile [3]. Based on the XPNAV Database, the X-ray pulsar signal photons' Joint Probability Density Function (JPDF) could be built [17]. By using the JPDF, we propose a Bayesian classifier for $\mathrm{X}$-ray pulsars recognition with the minimum recognition error criterion [18]. Unlike the traditional transforming method, the proposed Bayesian classifier could extract the features of pulsars without recovering the profile. In the condition of short observation time, the proposed Bayesian classifier shows high recognition efficiency.

This paper is organized as follows: Section 2 introduces the X-ray pulsar signal model; Section 3 explains the proposed Bayesian classifier in detail; Computer simulations for proving the classifier's validity are shown in Section 4, and some conclusions are given in the final part.

\section{X-Ray Pulsar Signal Model}

Unlike the radio signal, the $\mathrm{X}$-ray pulsar signal shows the particle property. The X-ray detector onboard aims at the 


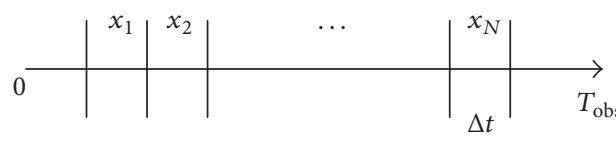

FIGURE 1: The segments of the observation time.

X-ray pulsar and records the arriving time of photons. The number of received photons is a stochastic process which obeys the nonhomogeneous Poisson process [17]; the Probability Density Function (PDF) is shown in

$$
P\left(N_{T_{\mathrm{obs}}}=k\right)=\frac{\left(\int_{0}^{T_{\mathrm{obs}}} \lambda(t) d t\right)^{k} \exp \left(-\int_{0}^{T_{\mathrm{obs}}} \lambda(t) d t\right)}{k !},
$$

where $T_{\mathrm{obs}}$ is the observation time, $N_{T_{\mathrm{obs}}}$ is the number of received photons, and $\lambda(t)$ is the arriving rate of the photons. $\lambda(t)$ is composed of the X-ray pulsar signal and the X-ray background noise:

$$
\lambda(t)=\lambda_{b}+\lambda_{s} h(\theta(t)),
$$

where $\lambda_{b}$ is the $\mathrm{X}$-ray background noise's rate which is almost time-invariant in the cosmos environment, $\lambda_{s}$ is the $\mathrm{X}$ ray pulsar signal's flux, $h(\theta(t))$ is the X-ray pulsar signal's normalization standard profile, and $\theta(t)$ is the X-ray pulsar signal's phase. As the X-ray pulsar signal is periodic, $h(\theta(t)+$ $T)=h(\theta(t))$, where $T$ is the period.

\section{Bayesian Classifier for \\ X-Ray Pulsar Recognition}

3.1. Classifier Description. Divide the observation time into $N$ segments, which is shown in Figure 1. Each segment time is $\Delta t$. If $\Delta t$ is short enough, the $\mathrm{X}$-ray photons' arriving rate in the $n$th segment $\lambda_{n}(\theta)$ could be expressed as [17]

$$
\lambda_{n}(\theta) \approx \frac{1}{\Delta t} \int_{t_{0}+(n-1) \Delta t}^{t_{0}+n \Delta t} \lambda(t ; \theta) d t
$$

The number of photons in the $n$th segment $x_{n}$ is a random variable which obeys the Poisson distribution. The PDF of $x_{n}$ could be expressed as

$$
p\left(x_{n}=k ; \theta\right)=\frac{\left(\lambda_{n}(\theta) \Delta t\right)^{k}}{k !} \exp \left(-\lambda_{n}(\theta) \Delta t\right) .
$$

As the random variables in each segment is independent, the Joint Probability Density Function (JPDF) could be expressed as

$$
\begin{aligned}
p(\mathbf{X} ; \theta) & =\prod_{n=1}^{N} p\left(x_{n} ; \theta\right) \\
& =\prod_{n=1}^{N} \frac{\left(\lambda_{n}(\theta) \Delta t\right)^{x_{n}}}{x_{n} !} \exp \left(-\lambda_{n}(\theta) \Delta t\right),
\end{aligned}
$$

where $\mathbf{X}=\left[\begin{array}{llll}x_{1} & x_{2} & \cdots & x_{N}\end{array}\right]^{\mathrm{T}}$.
In order to simplify the analysis, we suppose recognizing two X-ray pulsars. The JPDF of these X-ray pulsar signals could be, respectively, expressed as

$$
\begin{aligned}
& H_{1}: p_{1}\left(\mathbf{X} ; \theta_{n}\right) \\
& =\prod_{n=1}^{N} \frac{\left(\left(\lambda_{b}+\lambda_{s}^{1} h_{1}\left(\theta_{n}\right)\right) \Delta t\right)^{x_{n}}}{x_{n} !} \exp \left(-\left(\lambda_{b}+\lambda_{s}^{1} h_{1}\left(\theta_{n}\right)\right) \Delta t\right), \\
& H_{2}: p_{2}\left(\mathbf{X} ; \theta_{n}\right) \\
& =\prod_{n=1}^{N} \frac{\left(\left(\lambda_{b}+\lambda_{s}^{2} h_{2}\left(\theta_{n}\right)\right) \Delta t\right)^{x_{n}}}{x_{n} !} \exp \left(-\left(\lambda_{b}+\lambda_{s}^{2} h_{2}\left(\theta_{n}\right)\right) \Delta t\right) .
\end{aligned}
$$

Based on the Bayesian theory, the probability of the error recognition is [18]

$$
\begin{aligned}
p_{e}= & p\left(H_{1} \mid H_{2}\right) p\left(H_{2}\right)+p\left(H_{2} \mid H_{1}\right) p\left(H_{1}\right) \\
= & p\left(H_{2}\right) \int_{R_{1}} p\left(\mathbf{X} \mid H_{2}\right) d \mathbf{X} \\
& +p\left(H_{1}\right) \int_{R_{2}} p\left(\mathbf{X} \mid H_{1}\right) d \mathbf{X} \\
= & p\left(H_{2}\right) \int_{R_{1}} p\left(\mathbf{X} \mid H_{2}\right) d \mathbf{X} \\
& +p\left(H_{1}\right)\left(1-\int_{R_{1}} p\left(\mathbf{X} \mid H_{1}\right) d \mathbf{X}\right) \\
= & p\left(H_{1}\right) \\
& +\int_{R_{1}}\left\{p\left(H_{2}\right) p\left(\mathbf{X} \mid H_{2}\right)-p\left(H_{1}\right) p\left(\mathbf{X} \mid H_{1}\right)\right\} d \mathbf{X},
\end{aligned}
$$

where $R_{1}$ is the decision region of $H_{1}$ and $R_{2}$ is the decision region of $\mathrm{H}_{2}$.

Our goal is to minimize $p_{e}$. Then, we decide that $\mathbf{X}$ belong to $H_{1}$, if and only if

$$
p\left(H_{2}\right) p\left(\mathbf{X} \mid H_{2}\right)-p\left(H_{1}\right) p\left(\mathbf{X} \mid H_{1}\right)<0 .
$$

Assume $p\left(H_{1}\right)=p\left(H_{2}\right)$ and take logarithm to the two sides of inequality (8). Then, we could get the decision criterion:

$$
\begin{aligned}
& \ln \frac{p\left(\mathbf{X} \mid H_{1}\right)}{p\left(\mathbf{X} \mid H_{2}\right)}>0, \quad \mathbf{X} \in H_{1}, \\
& \ln \frac{p\left(\mathbf{X} \mid H_{1}\right)}{p\left(\mathbf{X} \mid H_{2}\right)} \leq 0, \quad \mathbf{X} \in H_{2} .
\end{aligned}
$$

Taking (6) into the proposed decision criterion (9),

$$
\begin{aligned}
& \ln \frac{p_{1}\left(\mathbf{X} ; \theta_{n}\right)}{p_{2}\left(\mathbf{X} ; \theta_{n}\right)}=\sum_{n=1}^{N}\left\{x _ { n } \left[\ln \left(\lambda_{b}+\lambda_{s}^{1} h_{1}\left(\theta_{n}\right)\right)\right.\right. \\
& \left.\left.-\ln \left(\lambda_{b}+\lambda_{s}^{2} h_{2}\left(\theta_{n}\right)\right)\right]\right\}-\sum_{n=1}^{N}\left\{\left[\lambda_{s}^{1} h_{1}\left(\theta_{n}\right)\right.\right. \\
& \left.\left.-\lambda_{s}^{2} h_{2}\left(\theta_{n}\right)\right] \Delta t\right\} .
\end{aligned}
$$


In (10), $\sum_{n=1}^{N}\left\{\left[\lambda_{s}^{1} h_{1}\left(\theta_{n}\right)-\lambda_{s}^{2} h_{2}\left(\theta_{n}\right)\right] \Delta t\right\}$ is constant in the condition of a fixed observation time. Assume $-\sum_{n=1}^{N}\left\{\left[\lambda_{s}^{1} h_{1}\left(\theta_{n}\right)-\lambda_{s}^{2} h_{2}\left(\theta_{n}\right)\right] \Delta t\right\}=W_{0}, \ln \left(\lambda_{b}+\lambda_{s}^{1} h_{1}\left(\theta_{n}\right)\right)-$ $\ln \left(\lambda_{b}+\lambda_{s}^{2} h_{2}\left(\theta_{n}\right)\right)=w_{n}$, and $\mathbf{W}=\left[\begin{array}{llll}w_{1} & w_{2} & \cdots & w_{N}\end{array}\right]^{\mathrm{T}}$, and then the classifier using the Bayesian minimum recognition error criterion for X-ray pulsar recognition could be expressed as

$$
g(\mathbf{X})=\mathbf{W}^{\mathrm{T}} \mathbf{X}+W_{0} .
$$

From (11), $g(\mathbf{X})=0$ determines a decision surface, and the surface is a hyperplane. The hyperplane divides the hyperspace into two parts, where $R_{1}=\{g(\mathbf{X}) \geq 0\}$ and $R_{2}=\{g(\mathbf{X})<0\}$.

3.2. Performance Estimation. The probability of the error recognition is shown in (7). In order to estimate $p_{e}$, we assume $p\left(H_{1}\right)=p\left(H_{2}\right)=0.5$, without generality, and calculate $\int_{R_{1}} p\left(\mathbf{X} \mid H_{2}\right) d \mathbf{X}$ and $\int_{R_{2}} p\left(\mathbf{X} \mid H_{1}\right) d \mathbf{X}$.

As $x_{n}$ obeys the Poisson distribution with the arriving rate $\lambda_{n}(\theta) \Delta t$, the PDF of $x_{n}$ is shown in (4). Then, the average of $x_{n}$ is $\varepsilon_{n}=E\left(x_{n}\right)=\lambda_{n}(\theta) \Delta t$ and the variance of $x_{n}$ is $\sigma_{n}^{2}=\operatorname{var}\left(x_{n}\right)=\lambda_{n}(\theta) \Delta t$. Each element of $\mathbf{X}$ is independent; based on the central limit theorem, $g(\mathbf{X})$ obeys the Gaussian distribution with the average $\varepsilon_{g(\mathbf{X})}$ and variance $\sigma_{g(\mathbf{X})}^{2}$. The PDF of $g(\mathbf{X})$ could be expressed as

$$
\begin{aligned}
P(g(\mathbf{X})) & =\frac{1}{\sqrt{2 \pi \sigma_{g(\mathbf{X})}}} \exp \left(-\frac{\left(g(\mathbf{X})-\varepsilon_{g(\mathbf{X})}\right)^{2}}{2 \sigma_{g(\mathbf{X})}^{2}}\right), \\
\varepsilon_{g(\mathbf{X})} & =E(g(\mathbf{X}))=E\left(\mathbf{W}^{\mathrm{T}} \mathbf{X}+W_{0}\right) \\
& =\sum_{n=1}^{N} w_{n} \varepsilon_{n}+W_{0}, \\
\sigma_{g(\mathbf{X})}^{2} & =\operatorname{var}(g(\mathbf{X}))=\operatorname{var}\left(\mathbf{W}^{\mathrm{T}} \mathbf{X}+W_{0}\right) \\
& =\sum_{n=1}^{N} w_{n}^{2} \sigma_{n}^{2} ;
\end{aligned}
$$

then,

$$
\begin{aligned}
& \int_{R_{2}} p\left(\mathbf{X} \mid H_{1}\right) d \mathbf{X}=P\left(g\left(\mathbf{X} \mid H_{1}\right)<0\right) \\
& \quad=\int_{-\infty}^{0} \frac{1}{\sqrt{2 \pi \sigma_{g\left(\mathbf{X} \mid H_{1}\right)}}} \exp \left(-\frac{\left(g\left(\mathbf{X} \mid H_{1}\right)-\varepsilon_{g\left(\mathbf{X} \mid H_{1}\right)}\right)^{2}}{2 \sigma_{g\left(\mathbf{X} \mid H_{1}\right)}^{2}}\right) d g \\
& \cdot\left(\mathbf{X} \mid H_{1}\right)=Q\left(\frac{g\left(\mathbf{X} \mid H_{1}\right)-\varepsilon_{g\left(\mathbf{X} \mid H_{1}\right)}}{\sigma_{g\left(\mathbf{X} \mid H_{1}\right)}}\right),
\end{aligned}
$$

where $Q(\cdot)$ is the $q$-function, $Q(x)=\int_{x}^{+\infty}(1 / \sqrt{2 \pi}) \exp (-(1 /$ 2) $\left.t^{2}\right) d t$.
The recognition probability of $H_{1}$ could be expressed as

$$
\begin{aligned}
P_{c}^{1} & =1-\int_{R_{2}} p\left(\mathbf{X} \mid H_{1}\right) d \mathbf{X} \\
& =1-Q\left(\frac{g\left(\mathbf{X} \mid H_{1}\right)-\varepsilon_{g\left(\mathbf{X} \mid H_{1}\right)}}{\sigma_{g\left(\mathbf{X} \mid H_{1}\right)}}\right) .
\end{aligned}
$$

Similarly, the recognition probability of $\mathrm{H}_{2}$ is

$$
\begin{aligned}
P_{c}^{2} & =1-\int_{R_{1}} p\left(\mathbf{X} \mid H_{2}\right) d \mathbf{X} \\
& =1-Q\left(\frac{g\left(\mathbf{X} \mid H_{2}\right)-\varepsilon_{g\left(\mathbf{X} \mid H_{2}\right)}}{\sigma_{g\left(\mathbf{X} \mid H_{2}\right)}}\right) .
\end{aligned}
$$

3.3. Recognition for Multiple X-Ray Pulsars. From (11), two $\mathrm{X}$-ray pulsars determine a decision surface. We expand the number of $\mathrm{X}$-ray pulsars to $K$; the number of decision surfaces is the combination $C_{K}^{2}$. To decrease the complexity of the Bayesian classifier, we deform (10) and calculate the features of each X-ray pulsar:

$$
\begin{aligned}
\ln & \frac{p_{1}\left(\mathbf{X} ; \theta_{n}\right)}{p_{2}\left(\mathbf{X} ; \theta_{n}\right)} \\
= & \sum_{n=1}^{N}\left\{x_{n} \ln \left(\lambda_{b}+\lambda_{s}^{1} h_{1}\left(\theta_{n}\right)\right)-\lambda_{s}^{1} h_{1}\left(\theta_{n}\right) \Delta t\right\} \\
& \quad-\sum_{n=1}^{N}\left\{x_{n} \ln \left(\lambda_{b}+\lambda_{s}^{2} h_{2}\left(\theta_{n}\right)\right)-\lambda_{s}^{2} h_{2}\left(\theta_{n}\right) \Delta t\right\} .
\end{aligned}
$$

The first item of (16) is only with a matter of the first Xray pulsar; the second item of (16) is only with a matter of the second X-ray pulsar. Then, we could get the features of the $i$ th $\mathrm{X}$-ray pulsar:

$$
J_{i}(\mathbf{X})=\sum_{n=1}^{N}\left\{x_{n} \ln \left(\lambda_{b}+\lambda_{s}^{i} h_{i}\left(\theta_{n}\right)\right)-\lambda_{s}^{i} h_{i}\left(\theta_{n}\right) \Delta t\right\} .
$$

By calculating $J(\mathbf{X})$ of every X-ray pulsar, the determined $\mathrm{X}$-ray pulsar is the one with the $\max \left(J_{i}(\mathbf{X})\right)$, where $\max (\cdot)$ is the maximum function.

3.4. Classifier with Unknown Parameters. As the spacecraft's position and the velocity are unknown in XPNAV, $\theta(t)$ in (2) would be influenced by the Doppler frequency $f_{d}$ and the initial phase $\phi_{0}$ :

$$
\begin{aligned}
\theta(t) & =\left(f_{0}+f_{d}\right) \cdot t+\phi_{0}, \\
f_{d} & =\frac{\mathbf{v} \cdot \mathbf{r}}{c} f_{0},
\end{aligned}
$$

where $f_{0}$ is the frequency of the X-ray pulsar, $\mathbf{v}$ is the spacecraft's velocity vector, $\mathbf{r}$ is the X-ray pulsar's direction vector, and $c$ is the light velocity. Before recognition, we have to evaluate $f_{d}$ and $\phi_{0}$ first. In this paper, we use the Maximum Likelihood (ML) estimation to deal with this 


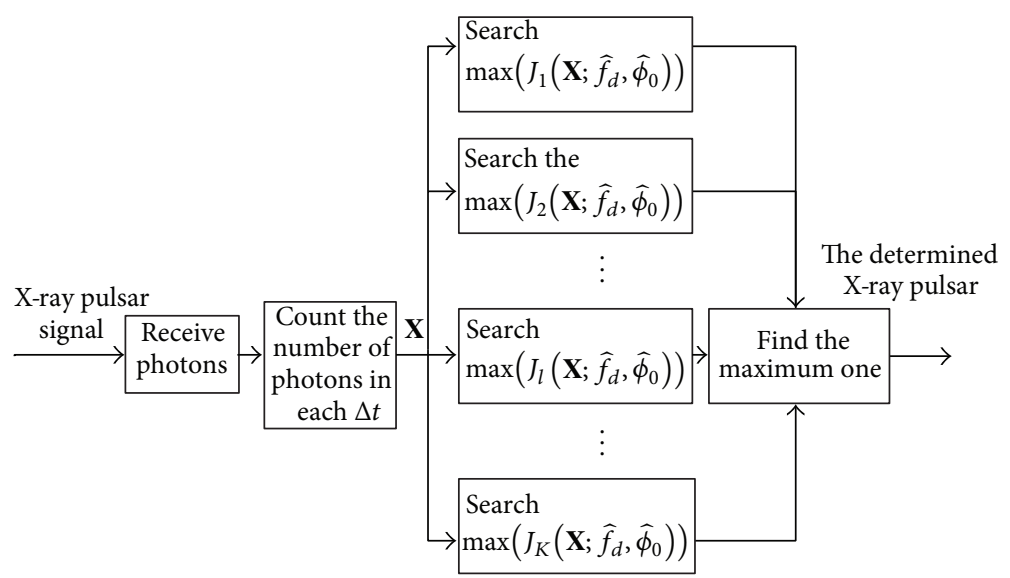

FIGURE 2: The whole recognition process for multiple X-ray pulsars with unknown parameters Bayesian classifier.

problem, because ML is asymptotic-efficient to the CramerRao Low Bound (CRLB) [17, 19].

Define $\max \left(J_{i}\left(\mathbf{X} ; \widehat{f}_{d}, \widehat{\phi}_{0}\right)\right)$ as the maximum of $J_{i}\left(\mathbf{X} ; \widehat{f}_{d}\right.$, $\left.\widehat{\phi}_{0}\right)$, and $\max \left(J_{i}\left(\mathbf{X} ; \hat{f}_{d}, \widehat{\phi}_{0}\right)\right)$ is selected as the features of the $i$ th X-ray pulsar for comparison, where $\widehat{f}_{d}$ is the evaluation of $f_{d}$ and $\widehat{\phi}_{0}$ is the evaluation of $\phi_{0}$. We do the two-dimensional grid searches for $f_{d}$ and $\phi_{0}$ to find the maximum value. The whole recognition process for multiple X-ray pulsars with unknown parameters Bayesian classifier is shown in Figure 2.

From (17), ignoring the computation burden for the part in $\{\cdot\}$, we need $N$ times additions for calculating the features of one X-ray pulsar. For $K \mathrm{X}$-ray pulsars, the computation is $K \times N$ times additions. If the searching interval of $f_{d}$ and $\phi_{0}$ is divided into $G$ grids. the whole number of additions is $G \times K \times N$.

3.5. Recognition for Unknown X-Ray Pulsars. There are hundreds of X-ray pulsars in universe. The XPNAV Database may only store some regular used X-ray pulsars' prior information. Therefore, we have to find a way to identify whether the received X-ray signal comes from the X-ray pulsars stored in the XPNAV Database or not.

From (17), $\left\{x_{n} \ln \left(\lambda_{b}+\lambda_{s}^{i} h_{i}\left(\theta_{n}\right)\right)-\lambda_{s}^{i} h_{i}\left(\theta_{n}\right) \Delta t\right\}$ obeys the Poisson distribution. When $N$ is large enough, $J_{i}(\mathbf{X})$ obeys the Gaussian distribution with the PDF:

$$
\begin{aligned}
& P\left(J_{i}(\mathbf{X})\right)=\frac{1}{\sqrt{2 \pi \sigma_{J_{i}(\mathbf{X})}}} \exp \left(-\frac{\left(J_{i}(\mathbf{X})-\varepsilon_{J_{i}(\mathbf{X})}\right)^{2}}{2 \sigma_{J_{i}(\mathbf{X})}^{2}}\right), \\
& \mathcal{E}_{J_{i}(\mathbf{X})}=E\left(J_{i}(\mathbf{X})\right) \\
& \quad=\sum_{n=1}^{N}\left\{\left(\lambda_{b}+\lambda_{s}^{i} h_{i}\left(\theta_{n}\right)\right) \ln \left(\lambda_{b}+\lambda_{s}^{i} h_{i}\left(\theta_{n}\right)\right)\right. \\
& \left.-\lambda_{s}^{i} h_{i}\left(\theta_{n}\right) \Delta t\right\}
\end{aligned}
$$

$$
\begin{aligned}
\sigma_{J_{i}(\mathbf{X})}^{2} & =\operatorname{var}\left(J_{i}(\mathbf{X})\right)=\operatorname{var}\left(\mathbf{W}^{\mathrm{T}} \mathbf{X}+W_{0}\right) \\
= & \sum_{n=1}^{N}\left\{\left(\lambda_{b}+\lambda_{s}^{i} h_{i}\left(\theta_{n}\right)\right)\left[\ln \left(\lambda_{b}+\lambda_{s}^{i} h_{i}\left(\theta_{n}\right)\right)\right]^{2}\right\} .
\end{aligned}
$$

We choose $\left(\varepsilon_{J_{i}(\mathbf{X})}-3 \sigma_{J_{i}(\mathbf{X})}, \varepsilon_{J_{i}(\mathbf{X})}+3 \sigma_{J_{i}(\mathbf{X})}\right)$ as the detection range of $J_{i}(\mathbf{X})$, as $P\left(\varepsilon_{J_{i}(\mathbf{X})}-3 \sigma_{J_{i}(\mathbf{X})}<J_{i}(\mathbf{X}) \leq \varepsilon_{J_{i}(\mathbf{X})}+3 \sigma_{J_{i}(\mathbf{X})}\right) \approx$ 99.7\%. If $J_{i}(\mathbf{X})$ is out of $\left(\varepsilon_{J_{i}(\mathbf{X})}-3 \sigma_{J_{i}(\mathbf{X})}, \varepsilon_{J_{i}(\mathbf{X})}+3 \sigma_{J_{i}(\mathbf{X})}\right)$, we judge that the received $\mathrm{X}$-ray signal comes from other $\mathrm{X}$-ray pulsars which are not included in the XPNAV Database or some Xray background noise in space.

\section{Computer Simulations}

4.1. Simulation for Two X-Ray Pulsars. Six X-ray pulsars from [7] are provided for Computer simulations. The parameters are shown in Table $1[3,7]$, and the normalization standard profiles are shown in Figure 3 [7, 20].

In order to certify the theory in Sections 3.1 and 3.2, we simulate the Bayesian classifier for two X-ray pulsars' $(\mathrm{B} 1821-24, \mathrm{~B} 1937+21)$ recognition. The time segment $\Delta t$ is set as $1 \mathrm{~ms}$ for simulation simplicity. In the condition of increasing observation time, we compare the recognition probability estimation from (14) with the 100 times Monte Carlo simulations. The photons generating algorithm refers to [17]. Simulation results are shown in Figures 4 and 5.

From Figures 4 and 5, the recognition probabilities increase with the increasing observation time. Besides that, the results of the Monte Carlo simulations are in accordance with the theory performance estimation from (14).

4.2. Simulation for Multiple X-Ray Pulsars. We simulate the Bayesian classifier for six X-ray pulsars' recognition with unknown parameters and without unknown parameters. The initial phase $\phi_{0}$ is set as a random number obeying the uniform distribution with the range $[0,1)$. The Doppler frequency $f_{d}$ obeys the uniform distribution with the range $(-0.0023,0.0023) \mathrm{Hz}$. The other simulation conditions are the same as those in Section 4.1. In the condition of increasing 
TABLE 1: The parameters of X-ray pulsars.

\begin{tabular}{|c|c|c|c|c|c|c|}
\hline ID & Name & $\begin{array}{c}\text { Period } \\
\text { (sec) }\end{array}$ & $\begin{array}{l}\text { Galactic longitude } \\
\text { (degree) }\end{array}$ & $\begin{array}{c}\text { Galactic latitude } \\
\text { (degree) }\end{array}$ & $\begin{array}{c}\lambda_{b} \\
\text { (cnts/s) }\end{array}$ & $\begin{array}{c}\lambda_{s} \\
\text { (cnts/s) }\end{array}$ \\
\hline 1 & B0531+21 & 0.0335 & 184.56 & -5.78 & 1540 & 15400 \\
\hline 2 & В0540-69 & 0.0504 & 279.72 & -31.52 & 5.15 & 51.5 \\
\hline 3 & B0833-45 & 0.0893 & 263.55 & -2.79 & 1.59 & 15.9 \\
\hline 4 & B1509-58 & 0.1502 & 320.32 & -1.16 & 16.2 & 162 \\
\hline 5 & B1821-24 & 0.0031 & 7.8 & -5.58 & 1 & 1.93 \\
\hline 6 & B1937+21 & 0.0016 & 57.51 & -0.29 & 1 & 0.499 \\
\hline
\end{tabular}
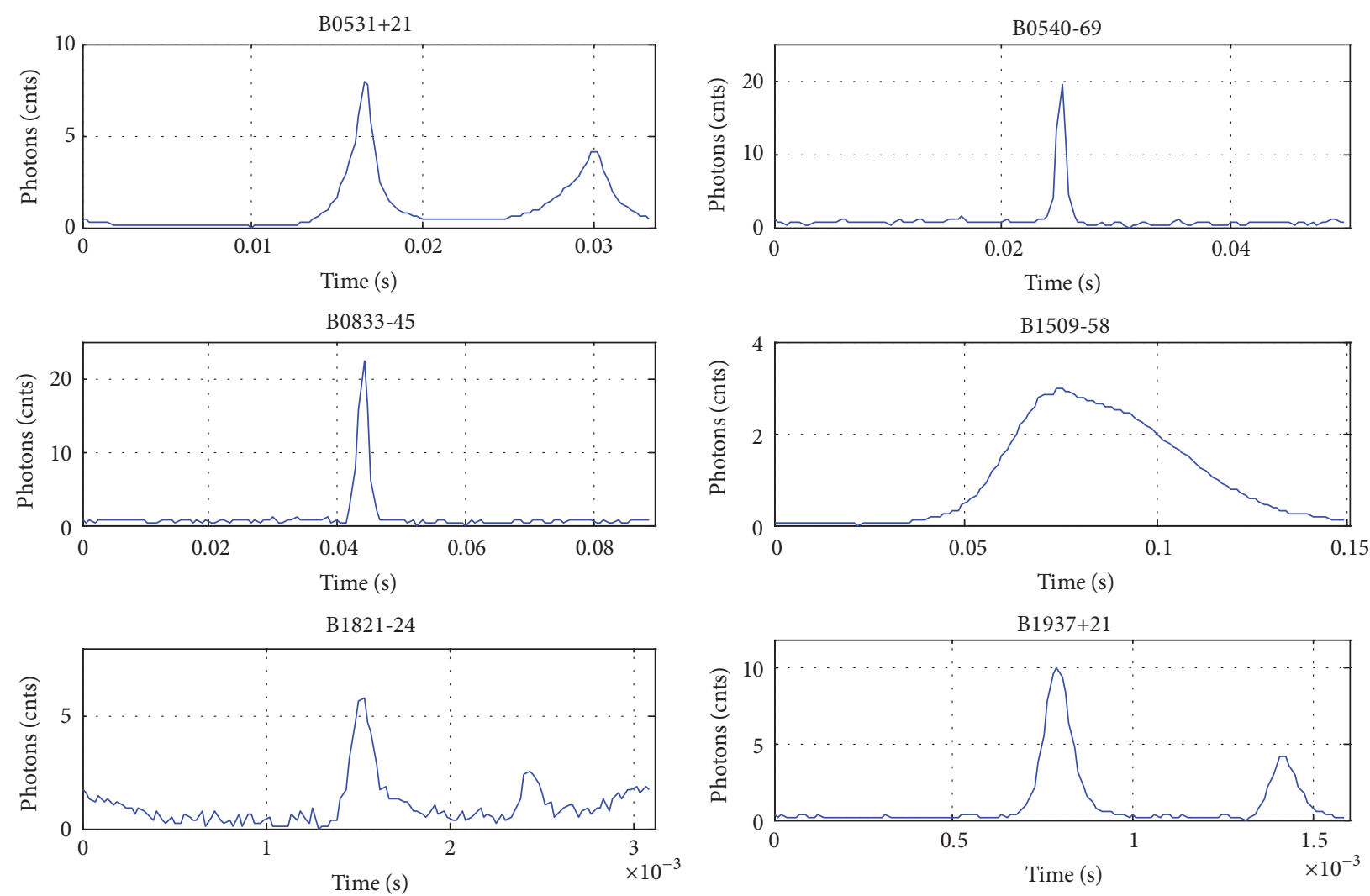

FIGURE 3: The normalization standard profile of six X-ray pulsars.

observation time, we simulate the recognition process presented in Figure 2. The searching range of $\phi_{0}$ is $[0,1)$, and the searching range of $f_{d}$ is $(-0.0023,0.0023) \mathrm{Hz}$. The searching step of $\phi_{0}$ is 0.02 , and the searching step of $f_{d}$ is $4.6 e-4 \mathrm{~Hz}$. The number of the Monte Carlo simulations is 100 times.

Besides that, as the ST method has both higher recognition rate and fast processing speed compared to the BispectraMellin Transform and the Wavelet Transform, we simulate the ST method for comparison [14]. The ST of the four Xray pulsars' normalization standard profiles are shown in Figure 6 . The detailed steps of the ST for X-ray pulsars recognition refer to $[13,14]$. The simulations for ST method ignore the impact of the unknown parameters for simplicity.

The recognition probability of each X-ray pulsar is shown in Figures 7-12.
From Figures 7-12, the recognition probabilities of six $\mathrm{X}$-ray pulsars utilizing the Bayesian classifier increase with the increasing observation time. Compared with the ST classifier, the Bayesian classifier obviously shows higher recognition probability, which certificates the effectiveness of the proposed Bayesian classifier. However, compared to the Bayesian classifier without unknown parameters, the one with unknown parameters shows lower recognition probabilities because of the limited estimation accuracy for $\phi_{0}$ and $f_{d}$.

The first four X-ray pulsars' (B0531+21, B0540-69, B0833$45, \mathrm{~B} 1509-58)$ recognition probabilities could reach $100 \%$ in no more than $1 \mathrm{~s}$, and the last two X-ray pulsars (B1821-24, $B 1937+21)$ need tens of seconds to get the same effect because of their lower flux. Therefore, we may select X-ray pulsars in 


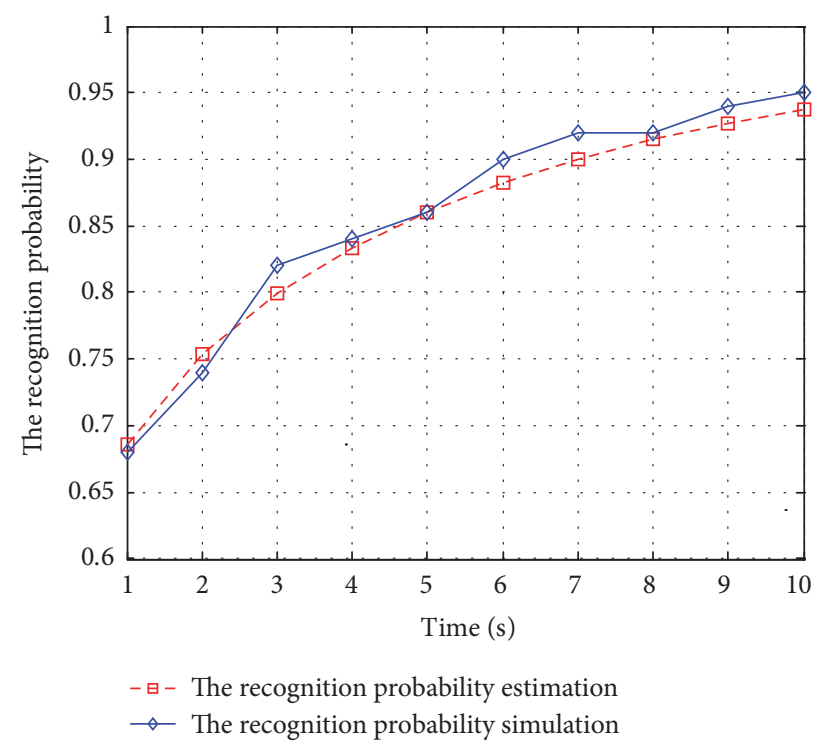

FIGURE 4: The recognition probability (two pulsars) for B1821-24.

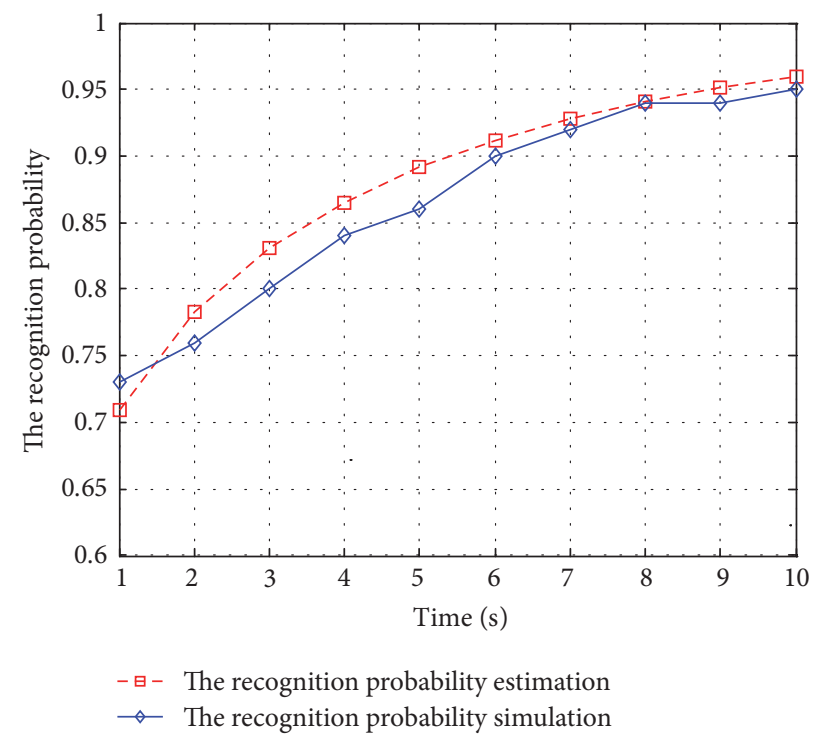

FIGURE 5: The recognition probability (two pulsars) for B1937+21.

the XPNAV Database with high flux for recognition and treat the X-ray pulsars with low flux as background noise.

4.3. Simulation for Unknown X-Ray Pulsars. We simulate the Bayesian classifier to recognize unknown X-ray pulsars. Two weak X-ray pulsars $(B 1821-24, B 1937+21)$ are selected as the unknown X-ray pulsars. Besides that, the signal only with background noise is also included in the simulation. We calculate $J_{i}(\mathbf{X}), \varepsilon_{J_{i}(\mathbf{X})}$, and $\sigma_{J_{i}(\mathbf{X})}^{2}$ of the remaining four X-ray pulsars in Table $1 . J_{i}(\mathbf{X})$ is calculated from $(17), \varepsilon_{J_{i}}(\mathbf{X})$ is calculated from (20), and $\sigma_{J_{i}(\mathbf{X})}^{2}$ is calculated from (21). If $J_{i}(\mathbf{X})$ is out of $\left(\varepsilon_{J_{i}(\mathbf{X})}-3 \sigma_{J_{i}(\mathbf{X})}, \varepsilon_{J_{i}(\mathbf{X})}+3 \sigma_{J_{i}(\mathbf{X})}\right)$, we judge that the received X-ray signal comes from other X-ray pulsars or $\mathrm{X}$-ray disturbances. The other simulation conditions are the same as those in Section 4.1. The number of the Monte Carlo simulations is 1000 .

The recognition probabilities of unknown X-ray pulsars are shown in Figures 13-15.

From Figures 13-15, the recognition probabilities for unknown X-ray pulsars or disturbance increase with the increasing observation time, and the recognition probabilities for two unknown X-ray pulsars and background noise could reach $100 \%$ in about $1.2 \mathrm{~s}$. Comparing Figures $7-10$ with $13-15,1.2 \mathrm{~s}$ is long enough for the first four X-ray pulsars' recognition process. 

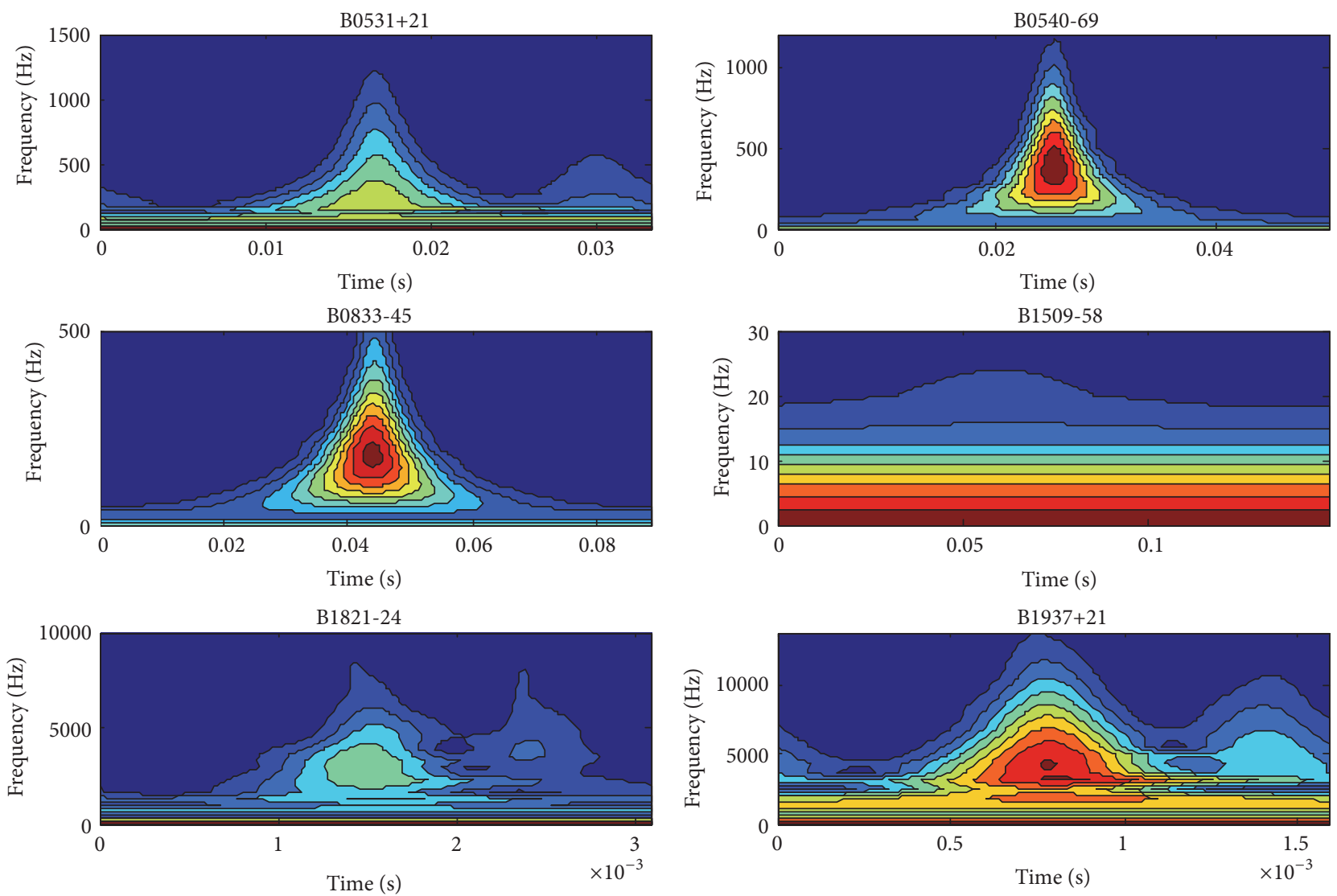

FIgURE 6: The S Transform for six X-ray pulsars.

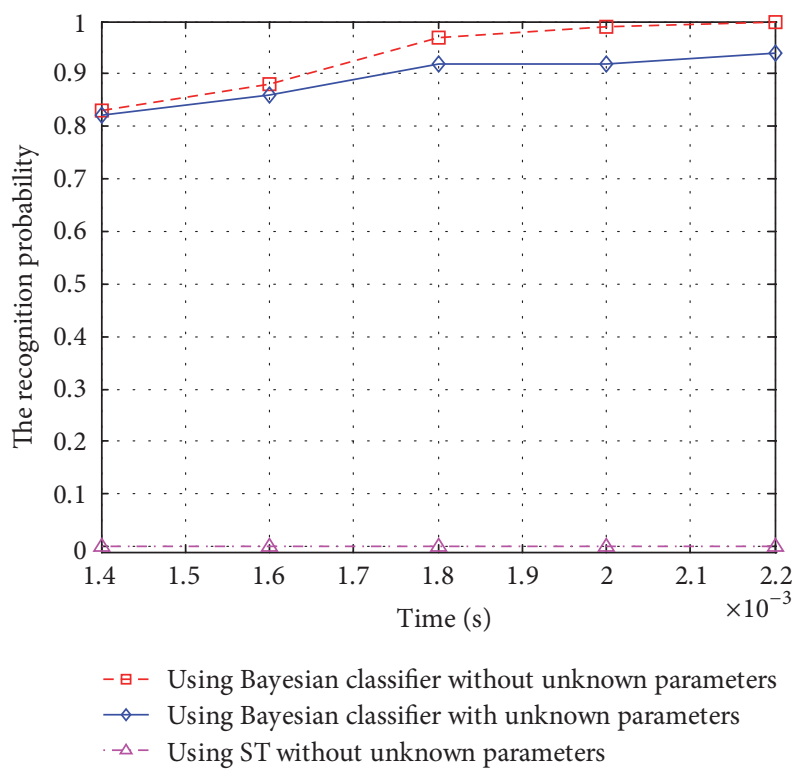

FIGURE 7: The recognition probability for B0531+21.

\section{Conclusion}

Recognition for X-ray pulsars is important in XPNAV system, especially for spacecraft's attitude determination. In this

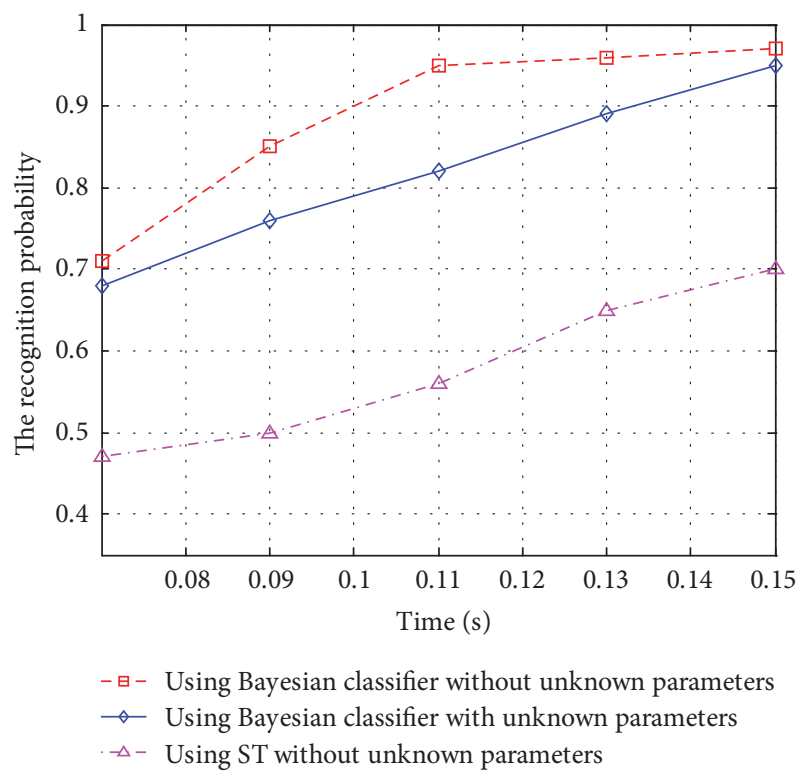

FIGURE 8: The recognition probability for B1509-58.

paper, we propose a decision surface of every two X-ray pulsars' recognition based on the Bayesian theory firstly and derive the recognition performance. Simulation results show that the theory performance estimations are in accordance 


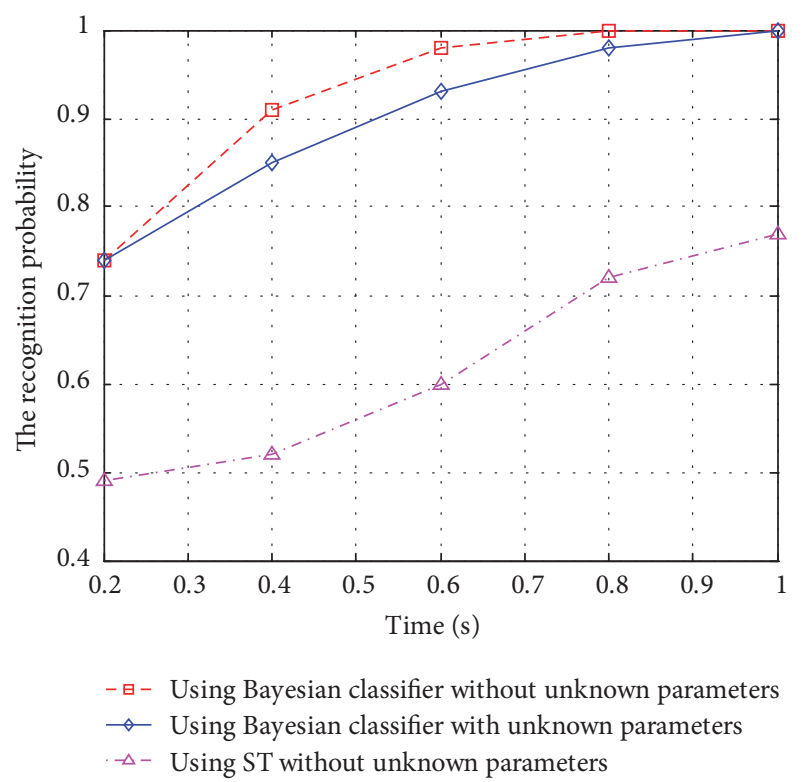

FIGURE 9: The recognition probability for B0833-45.

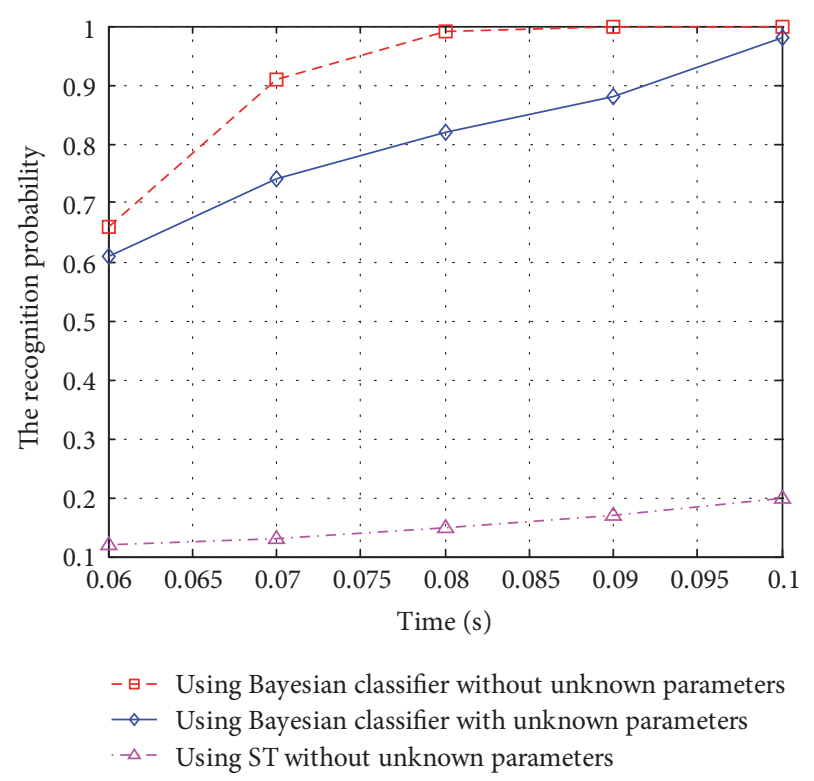

FIGURE 10: The recognition probability for B1509-58.

with the Monte Carlo simulations. We also extract the features of X-ray pulsars for multiple X-ray pulsars recognition and use the ML algorithm to estimate the unknown Doppler frequency and initial phase. Simulation results show that, in the condition of short observation time, the Bayesian classifier's recognition probability is obviously higher than that of the S Transform method. Besides that, we propose a method to recognize the unknown X-ray pulsars or X-ray disturbances. For some weak X-ray pulsars or background noise, the recognition time would be less than $1.2 \mathrm{~s}$, which could fulfill the practical requirements.

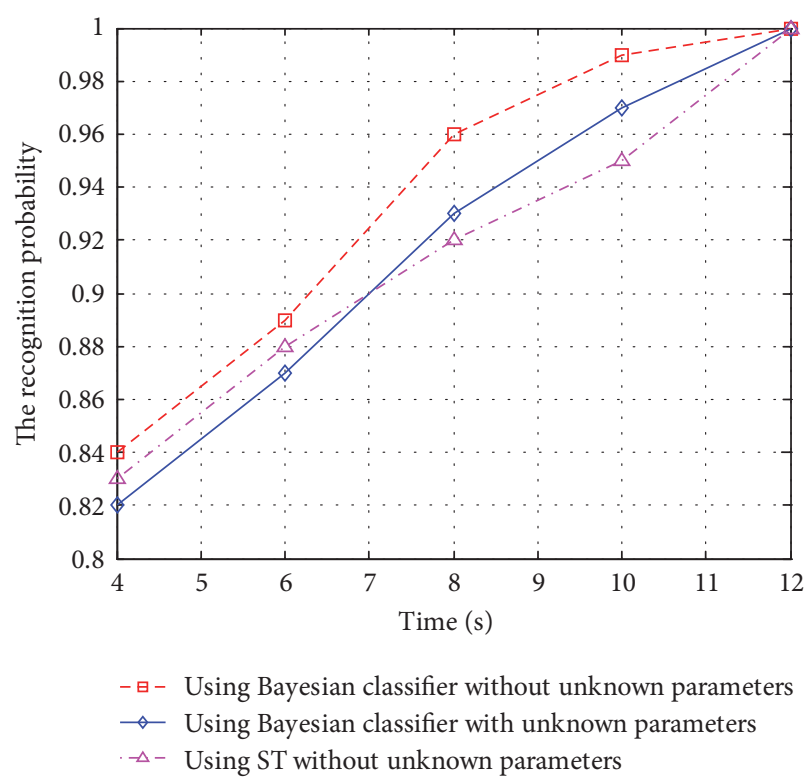

FIGURE 11: The recognition probability for B1821-34.

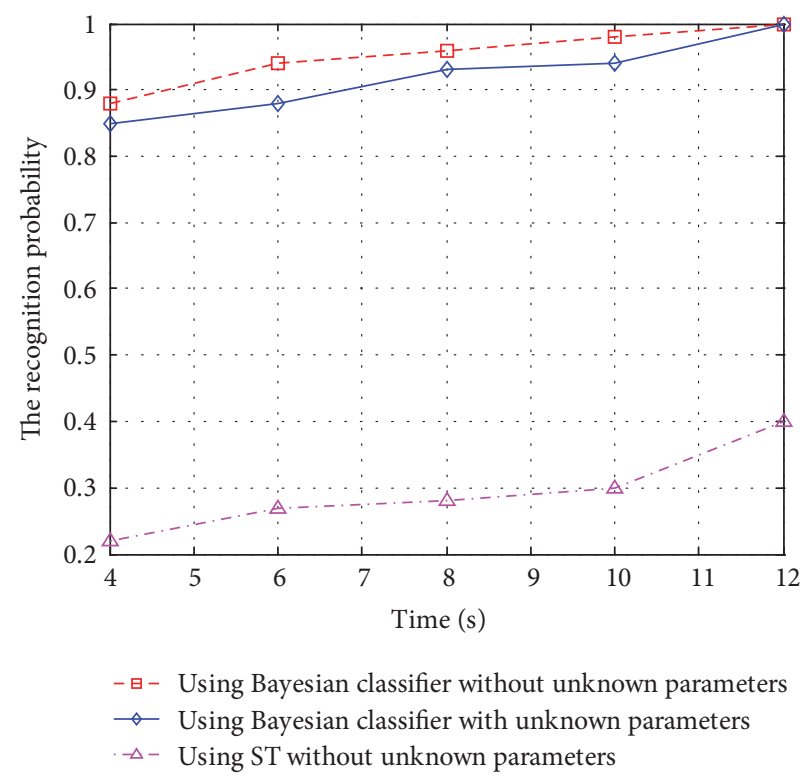

FIGURE 12: The recognition probability for B1937+21.

We believe the proposed Bayesian classifier may also be extended to other pattern recognition problems with the probability density obeying the Poisson distribution.

\section{Competing Interests}

The authors declare that they have no competing interests.

\section{Acknowledgments}

This work was supported in part by National Natural Science Foundation of China under Grants 61271265 and 


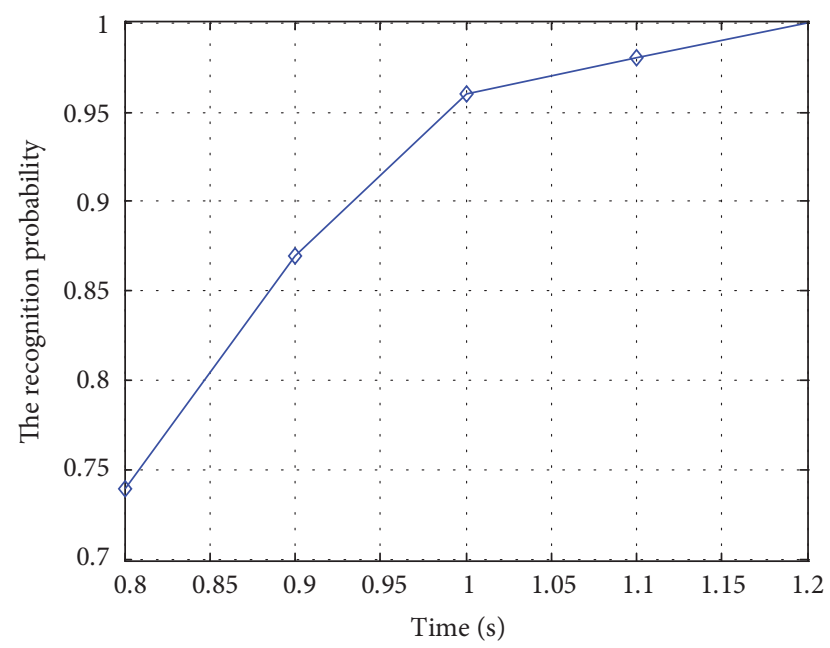

FIgURE 13: The recognition probability for unknown X-ray pulsars (B1821-24).

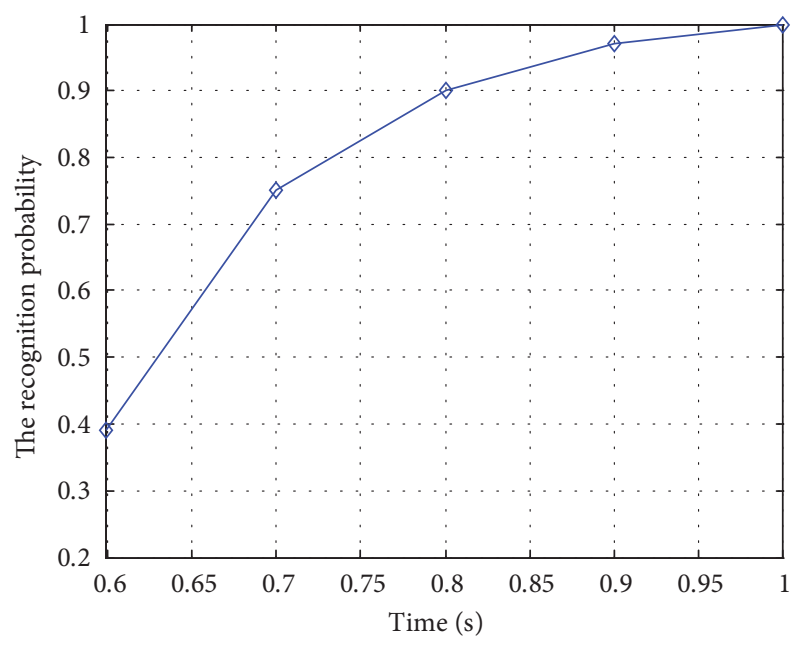

FIGURE 14: The recognition probability for unknown X-ray pulsars $(\mathrm{B} 1937+21)$.

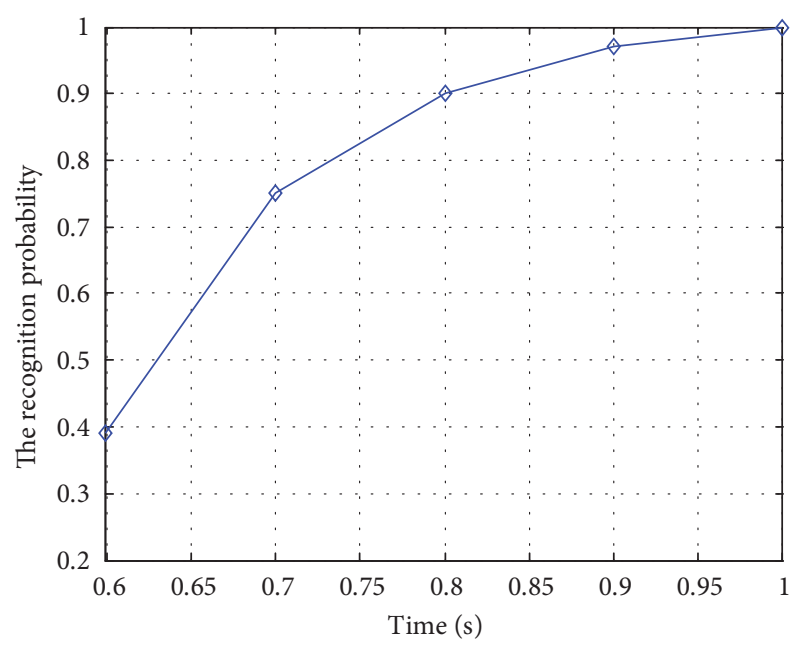

FIGURE 15: The recognition probability for unknown X-ray pulsars (background noise).
61671263 and in part by Tsinghua University Initiative Scientific Research Program under Grants 2013089244 and 20161080057.

\section{References}

[1] J. H. Taylor Jr., "Millisecond pulsars: Nature's most stable clocks," Proceedings of the IEEE, vol. 79, no. 7, pp. 1054-1062, 1991.

[2] D. N. Matsakis, J. H. Taylor, and T. M. Eubanks, "A statistic for describing pulsar and clock stabilities," Astronomy and Astrophysics, vol. 326, no. 3, pp. 924-928, 1997.

[3] S. I. Sheikh, The use of variable celestial X-ray sources for spacecraft navigation [Ph.D. dissertation], Department of Aerospace Engineering, University of Maryland, College Park, Md, USA, 2005.

[4] T. J. Chester and S. A. Butman, "Navigation using X-ray pulsars," Tech. Rep. 81N27129, JPL, NASA, Pasadena, Calif, USA, 1981.

[5] J. E. Hanson, Principles of X-ray navigation [Ph.D. thesis], Department of Aeronautics and Astronautics, Stanford University, Stanford, Calif, USA, 1996.

[6] S. I. Sheikh, D. J. Pines, P. S. Ray, K. S. Wood, M. N. Lovellette, and M. T. Wolff, "Spacecraft navigation using X-ray pulsars," Journal of Guidance, Control, and Dynamics, vol. 29, no. 1, pp. 49-63, 2006.

[7] A. A. Emadzadeh and J. L. Speyer, "Relative navigation between two spacecraft using X-ray pulsars," IEEE Transactions on Control Systems Technology, vol. 19, no. 5, pp. 1021-1035, 2011.

[8] S. I. Sheikh, J. E. Hanson, P. H. Graven et al., "Spacecraft navigation and timing using X-ray pulsars," Navigation, vol. 58, no. 2, pp. 165-186, 2011.

[9] D. Zhang, W. Zheng, Y. Wang, and L. Zhang, "X-ray pulsar profile recovery based on tracking-differentiator," Mathematical Problems in Engineering, vol. 2016, Article ID 4238165, 10 pages, 2016.

[10] X.-D. Zhang, Y. Shi, and Z. Bao, "A new feature vector using selected bispectra for signal classification with application in radar target recognition," IEEE Transactions on Signal Processing, vol. 49, no. 9, pp. 1875-1885, 2001.

[11] Z.-H. Xie, L.-P. Xu, G.-R. Ni, and Y. Wang, "A new feature vector using selected line spectra for pulsar signal bispectrum characteristic analysis and recognition," Chinese Journal of Astronomy and Astrophysics, vol. 7, no. 4, pp. 565-571, 2007.

[12] Z. Su, Y. Wang, L.-P. Xu, and N. Luo, "A new pulsar integrated pulse profile recognition algorithm," Journal of Astronautics, vol. 31, no. 6, pp. 1563-1568, 2010.

[13] R. G. Stockwell, L. Mansinha, and R. P. Lowe, "Localization of the complex spectrum: the S transform," IEEE Transactions on Signal Processing, vol. 44, no. 4, pp. 998-1001, 1996.

[14] L. Wang, L.-P. Xu, H. Zhang, and N. Luo, "A new algorithm of pulsar signal recognition," Journal of Astronautics, vol. 33, no. 10, pp. 1460-1465, 2012.

[15] H. Shan, X. Wang, X. Chen et al., "Wavelet based recognition for pulsar signals," Astronomy and Computing, vol. 11, pp. 5563, 2015.

[16] W. W. Zhu, A. Berndsen, E. C. Madsen et al., "Searching for pulsars using image pattern recognition," The Astrophysical Journal, vol. 781, no. 2, pp. 1-12, 2014.

[17] A. A. Emadzadeh and J. L. Speyer, "On modeling and pulse phase estimation of X-ray pulsars," IEEE Transactions on Signal Processing, vol. 58, no. 9, pp. 4484-4495, 2010. 
[18] A. R. Webb and K. D. Copsey, Statistical Pattern Recognition, John Wiley \& Sons, West Sussex, UK, 2011.

[19] N. Ashby and A. R. Golshan, "Minimum uncertainties in position and velocity determination using $\mathrm{x}$-ray photons from millisecond pulsars," in Proceedings of the ION NTM Conference, pp. 110-118, San Diego, Calif, USA, January 2008.

[20] The EPN (European Pulsar Network) Database Browser, http:// www.jb.man.ac.uk/ pulsar/Resources/epn/browser.html. 


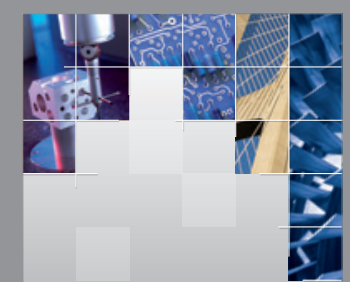

\section{Enfincering}
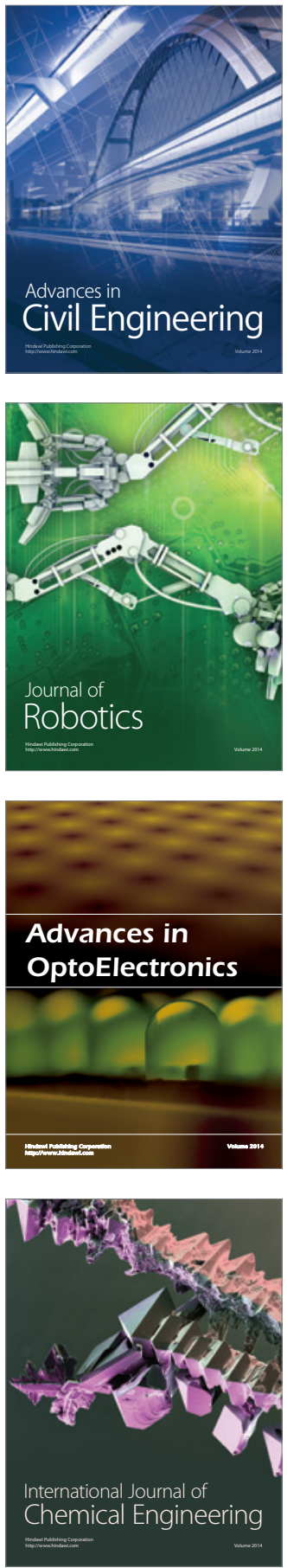

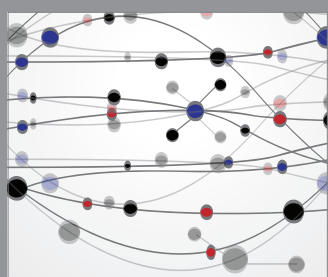

The Scientific World Journal

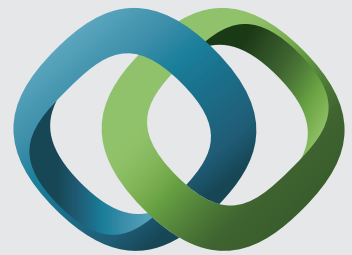

\section{Hindawi}

Submit your manuscripts at

http://www.hindawi.com
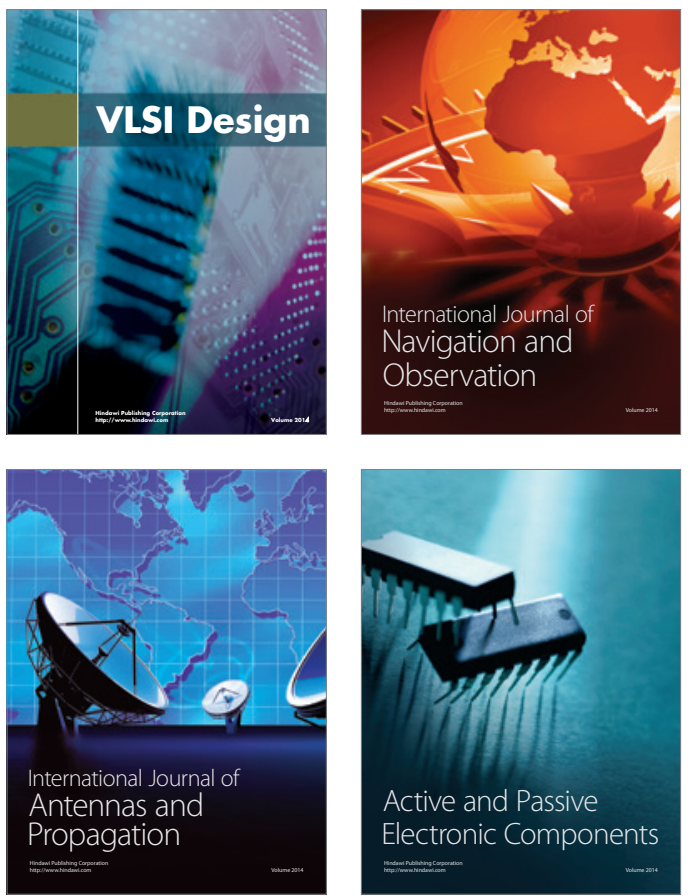
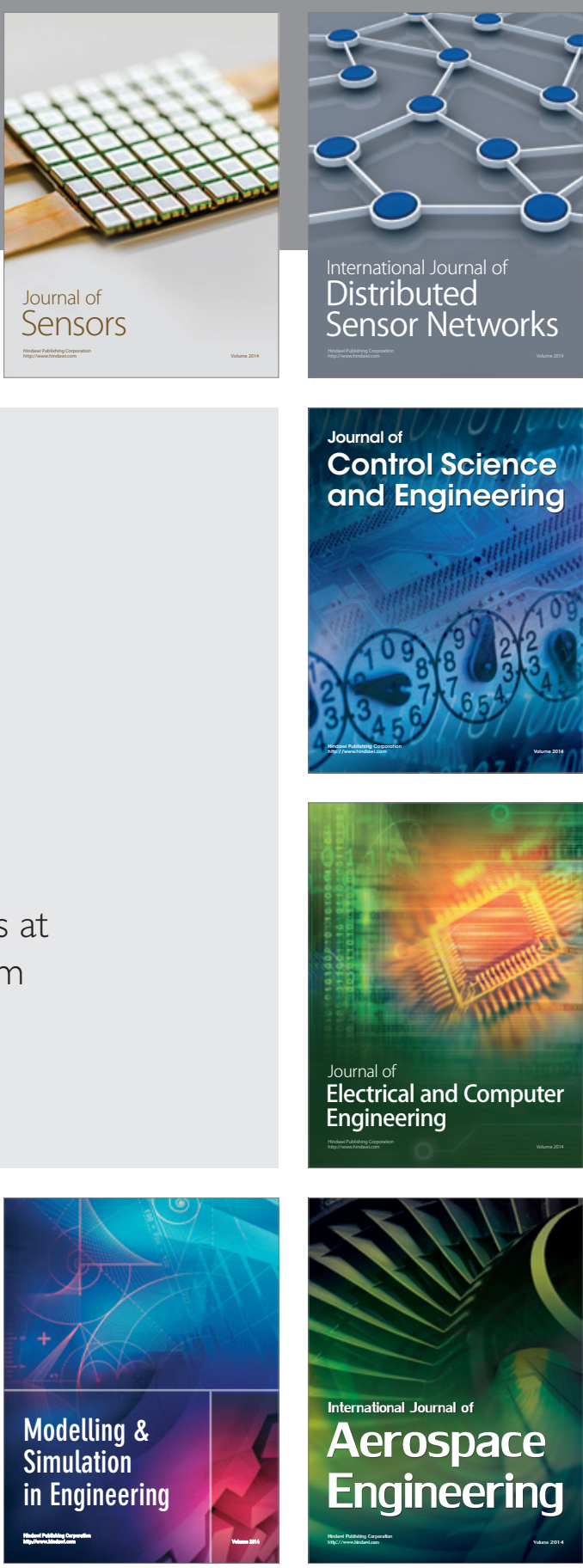

International Journal of

Distributed

Sensor Networks

Journal of

Control Science

and Engineering
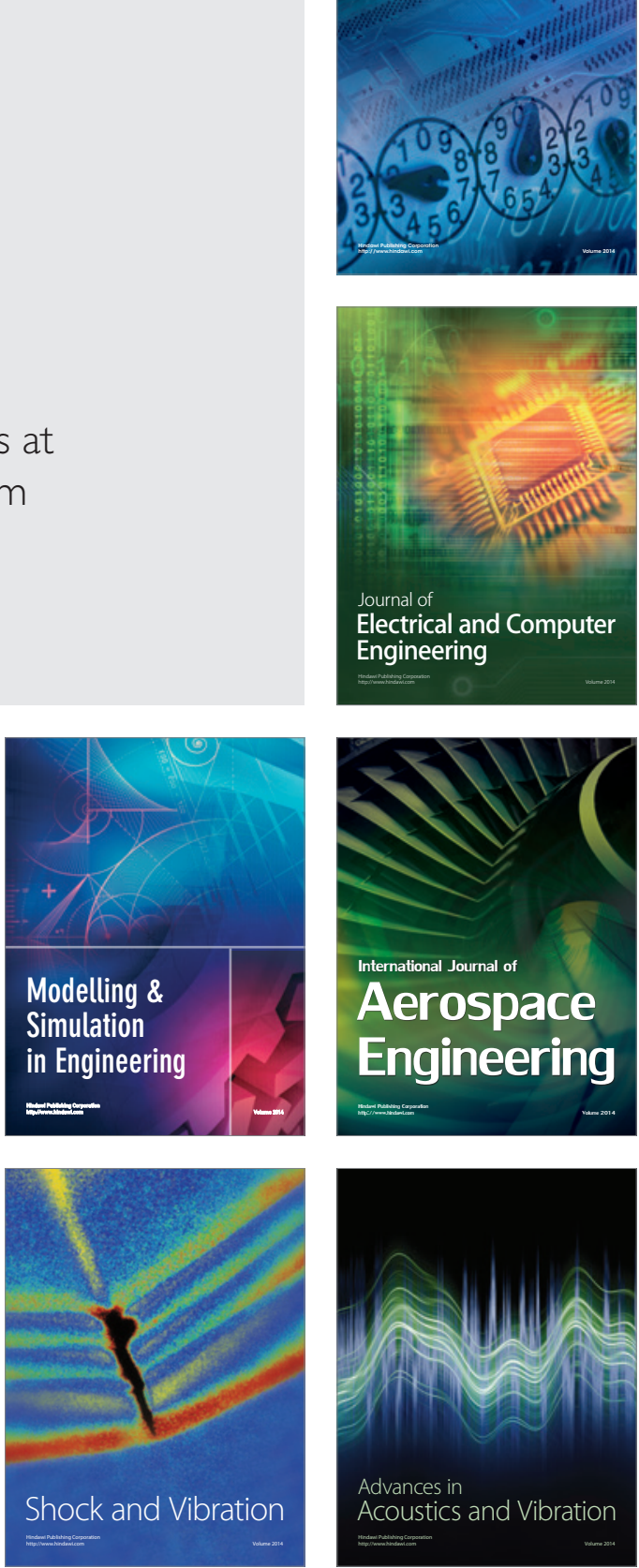